\title{
BMJ Open Disparity of the Chinese elderly's health-related quality of life between urban and rural areas: a mediation analysis
}

\author{
Xinyi You, ${ }^{1}$ Yali Zhang, ${ }^{1}$ Jinfeng Zeng, ${ }^{1}$ Congju Wang, ${ }^{2}$ Hongpeng Sun, ${ }^{1}$ \\ Qinghua Ma, ${ }^{3}$ Yana Ma, ${ }^{1}$ Yong $\mathrm{Xu}^{1}$
}

To cite: You X, Zhang Y, Zeng J, et al. Disparity of the Chinese elderly's healthrelated quality of life between urban and rural areas: a mediation analysis. BMJ Open 2019;9:e024080. doi:10.1136/ bmjopen-2018-024080

- Prepublication history and additional material for this paper are available online. To view these files, please visit the journal online (http://dx.doi org/10.1136/bmjopen-2018024080).

$X Y$ and $Y Z$ contributed equally.

Received 12 May 2018 Revised 4 November 2018 Accepted 18 December 2018

\section{Check for updates}

(c) Author(s) (or their employer(s)) 2019. Re-use permitted under CC BY-NC. No commercial re-use. See rights and permissions. Published by BMJ.

${ }^{1}$ Department of Child Health, Jiangsu Key Laboratory of Preventive and Translational Medicine for Geriatric Diseases, School of Public Health, Soochow University, Suzhou, China

${ }^{2}$ Department of Chronic Disease Management, Centers for Disease Control and Prevention of Suzhou High-tech Zone,

Suzhou, China

${ }^{3}$ Department of Chronic Disease Management, The 3rd People's Hospital of Xiangcheng District, Suzhou, China

Correspondence to

Dr Hongpeng Sun;

hpsun@suda.edu.cn

\section{ABSTRACT}

Objectives This study aimed to examine the urban-rural disparity in health-related quality of life (HRQoL) of the Chinese elderly and to explore the mediating roles of socioeconomic status (SES) and frequency of contact with children in the relationship between urban/rural areas and HRQoL.

Methods This cross-sectional study used data from China Health and Retirement Longitudinal Study for 2015-2016, involving 12369 Chinese aged 45 years and over. HRQDL of respondents was measured by three-level EuroQol five dimensions (EQ-5D-3L). SES, based on principal components analysis, was combined by the individual possessions of durable consumer goods and houses. Frequency of contact with children was derived from the responses to whether they live with children and how often they contact with them. Mediation analyses were performed to examine the mediating effects of SES and frequency of contact in the relationship between urban/ rural areas and $\mathrm{HRQOL}$.

Results Urban respondents had higher scores of $\mathrm{HRQOL}$ than rural respondents $(p<0.05)$. As SES and frequency of contact with children increased, the scores of HRQOL of the elderly went up as well. Mediation analyses proved the possible mediating effects of SES and frequency of contact in the relationship between urban/rural areas and HRQOL (0.0713 and 0.0064). The indirect effects induced by SES and frequency of contact were $65.45 \%$ and $5.90 \%$, respectively.

Conclusions There was a significant difference in HRQoL between urban and rural middle-aged and elderly participants, which was partially mediated by urban-rural disparities in SES and frequency of contact with children. Higher SES and frequency of contact with children contributed to higher health status in the Chinese elderly.

\section{INTRODUCTION}

With the rapid development of Chinese economy, life expectancy increased from 67.9 years in 1981 to 76.5 years in $2016,{ }^{1}$ people aged 65 years and over accounted for $8.87 \%$ of the total. ${ }^{2}$ However, the evaluation of the health of population should focus on the length of life and the health-related quality
Strengths and limitations of this study

- This study aimed to explore the causes of urban-rural disparity in health-related quality of life (HRQoL) among the middle-aged and elderly from two angles-SES and frequency of contact with children through multiple mediation analysis.

- This study employed principle component analysis, a more comprehensive method, to generate the mediator of 'SES' as a replacement of income or wealth.

- SES and frequency of contact with children only explained partial indirect effect, and the current study could not explain the whole mediating effects between urban-rural disparity and HRQOL.

of life (HRQoL) and its differences. A large number of studies have found that a huge disparity in HRQoL exist among urban and rural middle-aged and elderly adults, ${ }^{3-5}$ and the overall HRQoL of urban residents is better than that of rural residents. ${ }^{6}$ It may be due to the large gap of development between urban and rural areas in China. Some relevant factors may contribute to the difference of HRQoL between urban and rural residents, such as demographic factors, marital status, level of education, socioeconomic status (SES) ${ }^{78}$ and social support, especially frequency of contact with children, which acts as the core of social relationship. ${ }^{9} 10$ Some researchers found that SES had a positive impact on HRQoL in China, Korea and many other countries. ${ }^{11} 12$

It has been well demonstrated that weak social ties could result in physical and mental health problems among the elderly. ${ }^{13} 14$ The scope of the social network usually shrinks as the elderly grow older ${ }^{15}$ and elderly parents' relationship with children becomes a greater proportion of their social relationships. Especially in China, blood relations were emphasised by the traditional culture; relationships 
between children and elderly parents have usually been considered a core element of the family structure. ${ }^{16} 17$ Elderly people who got support from their family members had an enhanced quality of life (QoL) ${ }^{18}$ Some studies have proposed that older adults who are less connected to their children exhibit the higher risk of developing depression. ${ }^{19}$ However, urbanisation in recent years has led to an increase in the number of empty nesters in rural areas and weakening of family function in rural areas. ${ }^{20}$

Although it has been demonstrated that urban-rural areas, SES and frequency of contact with children have association with HRQoL of the middle aged and elderly, ${ }^{21}$ few studies has explored the mediating effects of SES and frequency of contact with children as mediators between urban-rural area and HRQoL of older adults in China. Therefore, we hope to study the possible mediating effect of SES and frequency of contact with children on the overall health status of older adults. Based on previous research, we hypothesise that: (1) Urban participants have higher level of HRQoL than rural participants, (2) Older adults with higher SES have higher HRQoL, (3) The participants who have more contacts with children may have higher HRQoL and (4) SES and frequency of contact with children may possibly mediate the difference of HRQoL between urban and rural elderly population.

\section{METHODS \\ Data}

Data were derived from the China Health and Retirement Longitudinal Study (CHARLS), which is a nationally representative longitudinal survey conducted by Institute of Social Science Survey, Peking University. Follow-up data were collected through face-to-face computer-assisted personal interview every 2years since 2011 to obtain a representative sample of Chinese aged 45 years and over in 28 provinces. A wide range of topics were involved in the questionnaire, including assessments of social, economic and health status of residents. This study used the third wave of CHARLS data for 2015-2016. In the first step, we selected 13920 respondents whose answers to questions related with HRQoL, SES and contact with their children were not missing. Then, after excluding missing values of other variables (age, gender, marital status and education attainment), a total of 12369 respondents with complete data on all variables were included in this analysis. Most baseline characteristics are comparable between enrolled and excluded respondents.

Our study followed the tenets of the Declaration of Helsinki. Written informed consent was obtained from all responders after the nature of the study was explained to them.

\section{Variables}

Dependent variable $(Y)$

HRQoL was measured by EQ-5D-3L ${ }^{22}$ that consists of a five-item descriptive system used to measure five dimensions of health status (mobility, self-care, usual activities, pain and depression) with three levels per domain (no problem, some problems and extreme problems). Two hundred and forty-three potential health states can generate in the form of a five-digit number, such as 23221, by combining every level of each dimension. In addition, the Chinese EQ-5D-3L value set based on time trade-off ${ }^{23}$ was used in order to elicit values of health states. Hence, the five-digit numbers of health status were all converted into numbers from -0.149 to 1 .

\section{Independent variable $(X)$}

The variable area of residency was geographically divided into urban or rural area on the basis of the National Bureau of Statistics of China. Value ' 1 ' represents the urban area and ' 0 ' represents the rural area.

\section{Mediators (M)}

The variable SES as a mediator represents the SES that can reflect the individual living standards. It is defined as a combination of economic, social and work status, which is commonly measured by income or wealth, education and occupation. Owing to the challenges in collecting income data, particularly in rural or low-income settings, the online supplementary information on income, such as household asset, can be used to form a composite asset index as a replacement of income or wealth. ${ }^{24}$ Principal components analysis (PCA) is a general method to create the index..$^{25}$ In this study, the synthetic asset index, based on PCA, was combined by the individual options about durable consumer goods (including automobile, electric bicycle, motorcycle, refrigerator, washing machine, $\mathrm{TV}$, computer, stereo system, video camera, camera, air conditioner, mobile phone, furniture, music instrument, valuable decorations, ornaments, treasures and precious metal and artistic work) and housing characteristics (including the type of structure of residence, one story or multilevel building, toilet, electricity, running water, bath facility, coal gas or natural gas, heating, source of cooking fuel, telephone and internet connection). Based on quintiles that are often used to be cut-off points for a given population, we classified the continuous index of SES into the following levels: $0=$ low, $1=$ middle low, $2=$ middle, $3=$ middle high and $4=$ high .

With regard to frequency of contact with children, whether the middle age and elderly had living children and cohabitated with their children were taken into consideration. Respondents without living children were considered the worst case, while those cohabitating with at least one child were divided into the group that had the most frequent contact with their children. Meanwhile, contact with children of those who had non-cohabitating children consists of two aspects that are 'face-to-face' contact and 'phone' contact. The conditions of contact with non-cohabitating children was described by the following questions in CHARLS: 'How often do you see your non-cohabiting children?' and 'How often do you have contact with children either by phone, text message, mail, or email when you didn't live with them?' The available options all ranged 


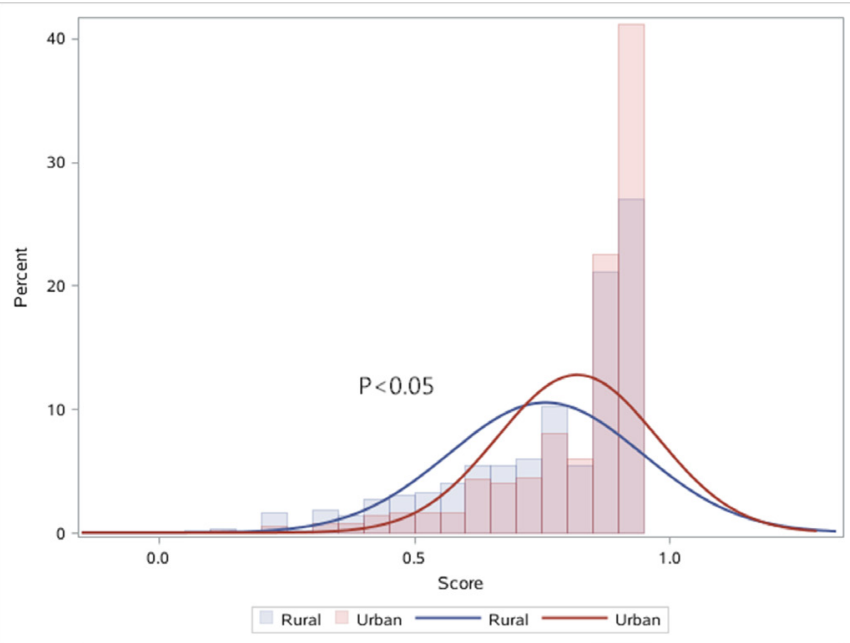

Figure 1 Urban/rural graphic distribution of the EQ-5D scale.

from ' $1=$ Almost every day' to ' $7=$ =almost never'. According to previous researches, ${ }^{19}$ we classified the frequency of contact with non-cohabitating children into four groups through the answers to these questions. Respondents in group $1 \mathrm{had}$ infrequent contact neither in face to face (less than once a month) nor phone way (less than once a week). Given that group 1 represented the least frequent contact, respondents without living children were in this group as well. Respondents in group 2 had frequent phone contact (once a week phone and more) but infrequent face-to-face contact (less than once a month). Respondents in group 3 had frequent face-to-face contact (once a month and more) but infrequent phone contact (less than once a week). Respondents in group 4 had both frequent face-to-face contact (once a month and more) and frequent phone contact (once a week and more). Since group 4 had the most frequency of contact, respondents cohabitating with their children were regarded as a part of this group as well.

\section{Other variables}

As the most relevant confounding factors, age, gender, marital status and education attainment were adjusted for in this study. Age was grouped into three classifications: 60 years and younger, 61-75 years and older than 75 years. Marital status was classified into six degrees including married and living with spouse, married but not living together temporarily, separated, divorced, widowed and never married. Education was classified into five degrees including illiterate/semiliterate, elementary school, junior high school, senior high school and college/university or higher.

\section{Statistical analysis}

Descriptive statistics were used to show the population distribution in demographic characteristics (age, gender, marital status and level of education), SES and frequency of contact between rural and urban respondents. The Kolmogorov-Smirnov test and the rank sum test were used to test the distribution and disparity of HRQoL between rural and urban areas. ${ }^{26}$ Stratified analysis was conducted for the variables (age, gender, marital status, education, SES and frequency of contact) to examine whether the disparity of HRQoL between rural and urban areas existed across strata.

Mediation analysis started with a simple mediation model (online supplementary figure 1.I) for SES and frequency of contact, respectively, which was used to verify the possible mediating effects of SES or frequency of contact on the relationship between area and HRQoL with a series of linear regression analysis. ${ }^{27}$ Besides, multiple mediation analysis was conducted in a singlestep multiple mediator model (online supplementary figure 1.II). ${ }^{28}$ This model simultaneously incorporated two mediators, which allowed to test an overall direct effect of area on HRQoL and to test specific indirect effect of one mediator while the other was controlled. In order to compare the magnitude of indirect effects of SES and contact, all variables were standardised according to suggestions from Mackinnon. ${ }^{29}$

In addition, magnitude of total, direct and indirect effect as well as 95\% CIs of indirect effect in aforementioned models were estimated based on a bootstrap method, which has been recommended as the most powerful and reasonable method to obtain estimation of indirect effects under most conditions that were not restricted to a normal sampling distribution. ${ }^{30}$ In this study, mediation analyses were computed from 5000 bootstrap samples. Mediating effects were also confirmed by the Sobel test, a statistical method that estimated the indirect effect and its SE. ${ }^{31}$ All analyses were performed using both SPSS software (V.20.0) with a process designed for mediation analysis ${ }^{32}$ and SAS software (V.9.4). The significance was set at $\alpha=0.05$. In aforementioned models, age, gender, marital status and education were controlled for all the time.

\section{Patient and public involvement statement}

CHARLS, as a household survey, involved no patients in the design or development of the research question and outcome measures. No patients were involved in developing the hypothesis and plans for design of this study either. The results would not be disseminated to study participants or any other individuals or communities.

\section{RESULTS}

\section{SES and frequency of contact with their children}

There were 12369 respondents in all. Compared with urban interviewees, rural interviewees were more likely to be male, older, lack of spouse companionship and undereducated. Besides these demographic differences, the elderly were generally poorer in rural areas. More than half $(52.43 \%)$ of the rural respondents were in middlelower and lower SES, while urban respondents in this status only accounted for $20.38 \%$ (online supplementary table 1).

Moreover, urban older adults contacted with their children more frequently (online supplementary table 2). 
After combination of two types of contact, the proportion of urban respondents in group 4 was significantly higher than that of rural respondents $(63.41 \%$ vs $44.59 \%)$. According to separate statistical data for frequency of two types of contact, urban respondents contacted with their children more frequently whatever the type was. Compared with urban respondents, rural older adults were more likely to have only one type of contact with children, either frequent face-to-face contact (group 3) or frequent phone contact (group 2).

\section{Health-related quality of life}

The graphic distribution of the EuroQol five dimensions (EQ-5D) scale shows a significant difference in HRQoL between urban and rural participants $(\mathrm{p}<0.05)$. Generally, the score of HRQoL of participants in urban areas was greater to that of rural areas (figure 1). Disparities between urban and rural areas in the score of HRQoL existed in all strata of gender, age and frequency of contact $($ all $\mathrm{p}<0.05)$. The result is given in the form of mean \pm SE. However, the statistical differences between urban and rural in the score of HRQoL disappeared when it comes to the elderly with senior high school or higher degree. Comparing the score of HRQoL in different marital status, the difference between urban and rural participants disappeared except for the level of 'married with spouse/living with partner', 'married but

Table 1 Stratified analysis of the score of HRQoL between urban and rural areas

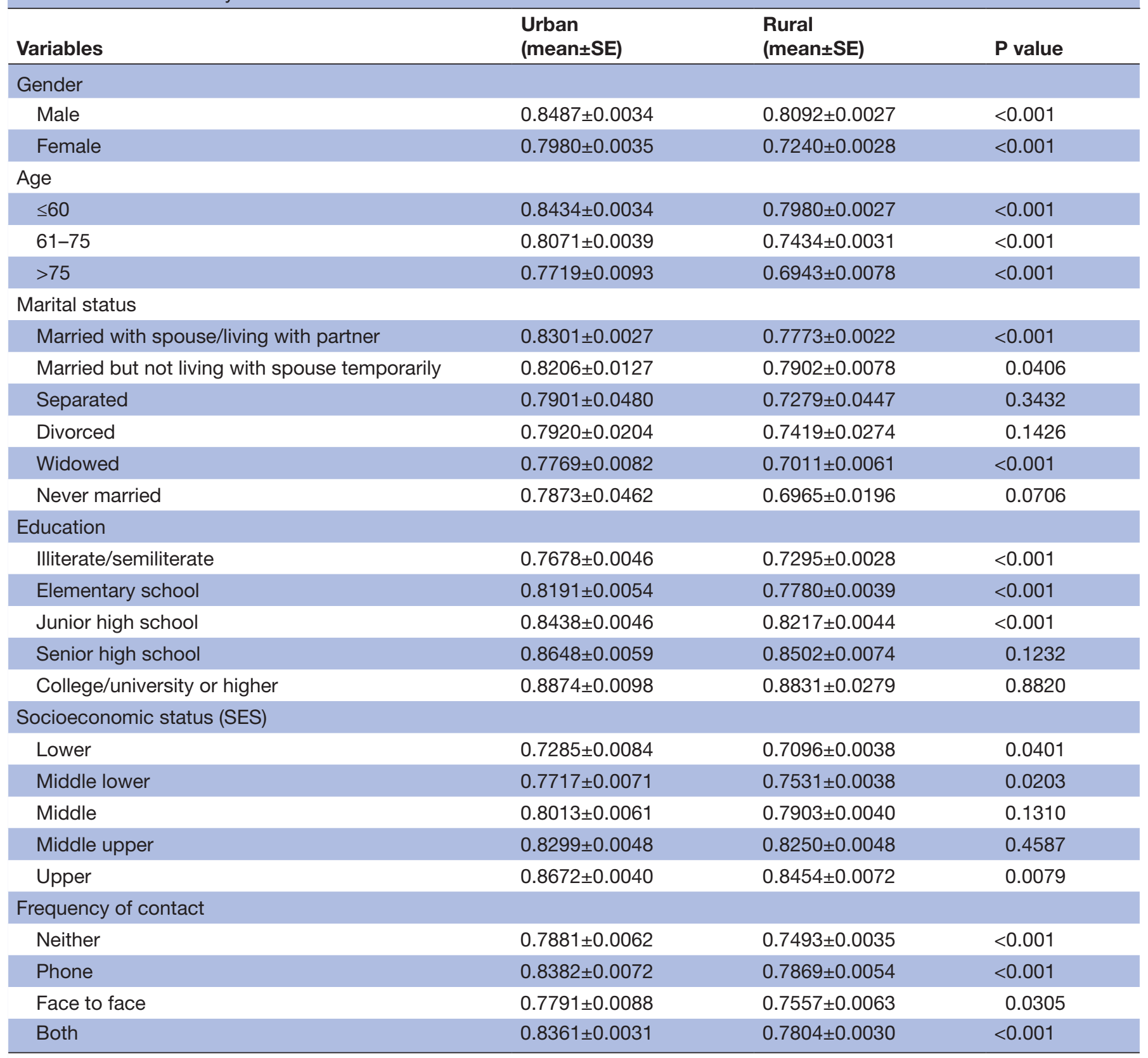

HRQoL, health-related quality of life. 
Table 2 The mediating effects of SES and contact on the relationship between area and HRQL in simple mediation model

\begin{tabular}{|c|c|c|c|c|c|}
\hline & Coefficient & SE & $P$ value & Sobel test: Z(P) & $\begin{array}{l}\text { Ratio of indirect } \\
\text { effect to total effect }\end{array}$ \\
\hline Area $\rightarrow$ SES $\rightarrow \mathrm{HRQoL}$ & & & & $19.3815(<0.0001)$ & $67.61 \%$ \\
\hline Direct effect $\left(c^{\prime}\right)$ & 0.0353 & 0.0094 & 0.0002 & & \\
\hline Area $\rightarrow$ SES (a) & 0.3488 & 0.0081 & $<0.0001$ & & \\
\hline Total effect (c path) & 0.1090 & 0.0089 & $<0.0001$ & & \\
\hline Direct effect $\left(c^{\prime}\right)$ & 0.0975 & 0.0090 & $<0.0001$ & & \\
\hline Area $\rightarrow$ contact $(\mathrm{a})$ & 0.1772 & 0.0093 & $<0.0001$ & & \\
\hline Contact $\rightarrow$ HRQoL (b) & 0.0651 & 0.0086 & $<0.0001$ & & \\
\hline
\end{tabular}

HRQoL, health-related quality of life; SES, socioeconomic status.

not living together temporarily' and 'widowed'. Similarly, the difference in the score of HRQoL between urban and rural middle-aged and elderly participants disappeared among the strata of SES for the level of middle and middle-upper SES. Moreover, as the educational level, SES and frequency of contact with children rose, scores of HRQoL of the elderly in both urban and rural areas went up (table 1).

\section{Mediation analyses}

The simple mediation analysis (table 2) indicated that area was positively associated with SES and HRQoL $(\mathrm{a}=0.3488$ and $\mathrm{c}=0.1090$, both $\mathrm{p}<0.0001)$. As a mediator, SES was positively associated with HRQoL $(b=0.2113$, $\mathrm{p}<0.0001)$, leading to a reduction in effect of area on HRQoL ( $c=0.1090$ vs $\left.c^{\prime}=0.0353\right)$. The percentage of indirect effect to total effect of area was $67.61 \%(\mathrm{Z}=19.3815$, $\mathrm{p}<0.0001)$. Moreover, frequency of contact with children had similar results as the mediating variable. Area was statistically significant related with frequency of contact and HRQoL $(\mathrm{a}=0.1772$ and $\mathrm{c}=0.1090$, both $\mathrm{p}<0.0001)$ and the frequency of contact was positively associated

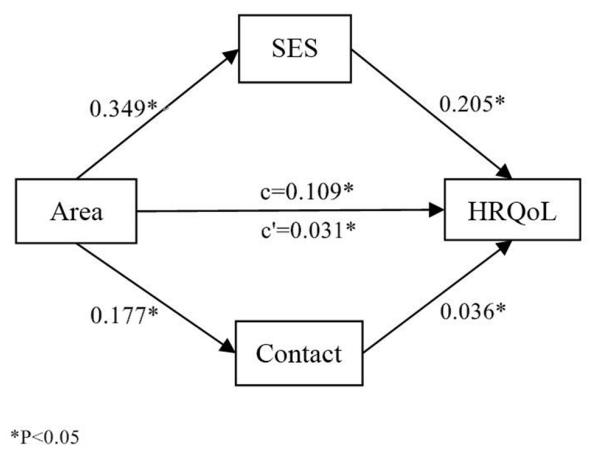

Figure 2 Multiple mediation analysis. HRQoL, health-related quality of life; SES, socioeconomic status. with HRQoL ( $b=0.0651, p<0.0001)$, explaining $10.59 \%$ of the variance in HRQoL induced by area $(\mathrm{Z}=7.0208$, $\mathrm{p}<0.0001)$.

Multiple mediation analysis showed that area, SES and frequency of contact with children were all related with HRQoL, and area had association with SES and frequency of contact as well (figure 2). Table 3 showed that the total effect of area on HRQoL was statistically significant and after adjusting for two mediators at the same time, the direct effect was still significant $\left(c=0.1090 \mathrm{vs} c^{\prime}=0.0312\right.$, both $\mathrm{p}<0.05)$. The mediating effects of SES and frequency of contact with children proved to be true with bootstrap CIs that did not contain zero (95\% CI 0.0637 to $0.0792 \%$ and $95 \%$ CI 0.0034 to 0.0097$)$. Besides, there was contrast between SES and frequency of contact with children that SES had a greater indirect effect in the association of area with HRQoL than frequency of contact $(65.45 \%$ vs $5.90 \%)$.

\section{DISCUSSION}

HRQoL, a comprehensive index, is selected as the independent variable to evaluate and compare the health status of the middle aged and elderly in both urban and rural areas. Our results explicitly reveal a huge gap, even after controlling known confounders, in the HRQoL among urban and rural individuals who are aged over 45 years and indicate that living in urban areas leads to better health status of older adults than rural areas. Moreover, the current study provides vital information about the relationship of urban-rural disparity in HRQoL with SES and frequency of contact with children among the middle aged and elderly in China. SES and frequency of contact with children play positive mediating roles in the difference of HRQoL between urban and rural older residents.

The mediation analysis indicates that the difference of HRQoL between urban and rural areas is partly mediated 
Table 3 The mediating effects of SES and contact on the relationship between area and HRQoL in single-step multiple mediator model

\begin{tabular}{|c|c|c|c|c|c|}
\hline & Parameter estimate & SE & $\begin{array}{l}\text { Lower } 95 \% \\
\text { BC Cl }\end{array}$ & $\begin{array}{l}\text { Upper } 95 \% \\
\text { BC CI }\end{array}$ & $\begin{array}{l}\text { Ratio of indirect effect to } \\
\text { total effect (\%) }\end{array}$ \\
\hline Total effect (c) & $0.1090^{*}$ & 0.0089 & 0.0916 & 0.1265 & \\
\hline Direct effect $\left(c^{\prime}\right)$ & $0.0312^{*}$ & 0.0094 & 0.0128 & 0.0497 & \\
\hline Indirect effect 1 (SES) & $0.0713^{*}$ & 0.0039 & 0.0637 & 0.0792 & 65.45 \\
\hline Indirect effect 2 (contact) & $0.0064^{*}$ & 0.0016 & 0.0034 & 0.0097 & 5.90 \\
\hline
\end{tabular}

${ }^{*} \mathrm{P}<0.05$

$\mathrm{X}^{2}=361.89(\mathrm{p}<0.0001)$ RMSEA: $0.171, \mathrm{GFI}: 0.858$ and NFI: 0.917 .

$\mathrm{BC} \mathrm{Cl}$, bias-corrected confidence intervals.

by SES. According to our analysis, families or individuals living in rural areas have lower level of SES, which will correspondingly contribute to the significantly worse self-perceived HRQoL of the middle-aged and elderly than those in cities. As shown in previous studies, the disparity mediated by SES may credit to the correlation between the level of health self-management and consciousness of the middle aged and elderly. ${ }^{33}$ Many studies have shown that high SES is indeed an advantage in promoting healthy behaviours and health service utilisation that further promote health status. ${ }^{34-36}$ Therefore, it is necessary for improving well-being of Chinese older adults to accelerate the process of balanced economic development and to enhance the equity of health service utilisation between urban and rural areas.

Another discovery of this study is that the frequency of contact with children plays an intermediary role in the relationship between urban/rural areas and HRQoL. We found that urban middle-aged and elderly residents have more contact with children than rural ones. The more frequent contact with children elderly residents have, the higher the index score of HRQoL they received. Considering the stage of China country's social development and demographic transition related with population policy, it may be ascribed to the migration to cities of many rural young workers for a better life. ${ }^{37}$ Rural-tourban migration is an important reason that decreases the frequency of contact between children and parents. The previous researches put forward that the elderly's social network affects their physical and psychological well-being by buffering stress and promoting health relevant behaviours. ${ }^{38-40}$ Meanwhile, other researches also stand the point that family and social support can affect the mental health of the elderly, children could play a key role in positively affecting the social network of their elderly parents ${ }^{15}$ and among elderly population, the risk of suffering from depression was negatively correlated with frequency of contact with adult children. ${ }^{19}$ Compared with the group of frequent face-to-face and phone contact with adult children, the risk of suffering from depression was higher in the other three groups, which lack of either face-to-face contact or phone contact or both. From the above, young people should give more care and support to the elderly. Meanwhile, the government should also encourage more young people to return to work in the countryside and support the development of rural economy in our country.

However, SES and frequency of contact with children merely explained partial disparity in HRQoL of urban and rural elderly participants in multiple mediation analysis. This may remind us that there are still many other mediation factors to be explored. Concerning the two mediators we studied, SES has greater mediating effect on the relationship between urban-rural disparity in HRQoL of middle-aged and elderly participants compared with frequency of contact. It is possible that SES as a synthetic index covers more extensively. In some ways, it is suggested that reducing the unfairness of economic development between urban and rural areas is the most important task for the country to improve the national health.

The current study has three major limitations. First, we employed the cross-sectional data that cannot distinguish cause and effect between mediators and HRQoL. The longitudinal mediation analysis will be a good choice in the future study. Second, the two mediators we chose only explained about $70 \%$ indirect effect, and the current study is not to further explore the relationship between the mediators and HRQoL. Some other researchers found the positive effects of SES on HRQoL of older adults might credit to some additional factors such as social functioning. Maybe a more complex mediation model or more mediation variables could be applied to explore the relevant relationship. Third, urban and rural respondents may have different performance in different dimensions of HRQoL. ${ }^{41}$ Further investigation could be conducted in each dimension separately to study the mediating effects of SES and frequency on urban-rural disparity among middle-aged and elderly people.

Our findings prove the fundamental influence of SES on health status of the middle aged and elderly. Therefore, this study is of great significance for the government to carry out future healthcare policies and promote the health equality for middle-aged and elderly residents in urban and rural areas. ${ }^{42}$ Moreover, it also provides the adequate foundation that young people need to provide financial support for our parents and to establish close relationships with them to maintain their QoL when they get older. 
Acknowledgements We would like to acknowledge the CHARLS team for the publicly available data.

Contributors HS and $Q M$ designed the experiments; $X Y$ and $Y Z$ collected the samples; $Y Z$ and JZ managed statistical analysis and interpretation of the results; $\mathrm{XY}$ wrote the manuscript; CW, YM and YX offered advice for study and helped language editing; all authors reviewed the manuscript.

Funding This study was funded by National Natural Science Foundation of China (81402761), the Foundation Research Project of Jiangsu Province (BK20140361), Suzhou Xiangcheng district people's livelihood science and technology project (XJ201655 and XJ201706).

Competing interests None declared.

Patient consent for publication Obtained.

Ethics approval Our study was approved by the ethics committee of the Medical College of Soochow University in Suzhou, Jiangsu, China.

Provenance and peer review Not commissioned; externally peer reviewed.

Data sharing statement The data sets used and analysed in this study are available from the China Health and Retirement Longitudinal Study (http://charls. pku.edu.cn/zh-CN), a nationally representative longitudinal survey conducted by Institute of Social Science Survey, Peking University.

Open access This is an open access article distributed in accordance with the Creative Commons Attribution Non Commercial (CC BY-NC 4.0) license, which permits others to distribute, remix, adapt, build upon this work non-commercially, and license their derivative works on different terms, provided the original work is properly cited, appropriate credit is given, any changes made indicated, and the use is non-commercial. See: http://creativecommons.org/licenses/by-nc/4.0/.

\section{REFERENCES}

1. China, T.s.c.o.t.P.s.R.o. Development of china's public health as an essential element of human rights. 2017 http://www.scio.gov.cn/ztk/ dtzt/36048/37159/37161/Document/1565159/1565159.htm (cited 14 April 2018).

2. China, N.b.o.s.o.t.P.s.R.o. The six national census. 2011 http:// www.stats.gov.cn/ztjc/zdtjgz/zgrkpc/dlcrkpc/dcrkpcyw/201104/ t20110428 69407.htm (cited 14 April 2018).

3. Tsai SY, Chi LY, Lee LS, et al. Health-related quality of life among urban, rural, and island community elderly in Taiwan. J Formos Med Assoc 2004;103:196-204.

4. Zhang Y. Research on the health related quality of life of Chinese residents. Medicine and Society 2013.

5. Oguzturk O. Differences in quality of life in rural and urban populations. Clin Invest Med 2008;31:346.

6. Dong $X$, Simon MA. Health and aging in a Chinese population: urban and rural disparities. Geriatr Gerontol Int 2010;10:85-93.

7. Lubetkin El, Jia H, Franks $\mathrm{P}$, et al. Relationship among sociodemographic factors, clinical conditions, and health-related quality of life: examining the EQ-5D in the U.S. general population. Qual Life Res 2005;14:2187-96.

8. Bobak M, Pikhart H, Rose R, et al. Socioeconomic factors, material inequalities, and perceived control in self-rated health: crosssectional data from seven post-communist countries. Soc Sci Med 2000;51:1343-50.

9. Liu LJ, Guo Q. Loneliness and health-related quality of life for the empty nest elderly in the rural area of a mountainous county in China. Qual Life Res 2007;16:1275-80.

10. Huguet N, Kaplan MS, Feeny D. Socioeconomic status and health-related quality of life among elderly people: results from the Joint Canada/United States Survey of Health. Soc Sci Med 2008;66:803-10.

11. Wu L, Zhang H. Health-related quality of life of low-socioeconomicstatus populations in Urban China. Health Soc Work 2016;41:219-27.

12. Kim HJ, Park S, Park SH, et al. The significance of frailty in the relationship between socioeconomic status and healthrelated quality of life in the Korean community-dwelling elderly population: mediation analysis with bootstrapping. Qual Life Res 2017;26:3323-30.

13. Kim HK, Hisata M, Kai I, et al. Social support exchange and quality of life among the Korean elderly. J Cross Cult Gerontol 2000;15:331-47.
14. Årestedt $\mathrm{K}$, Saveman $\mathrm{BI}$, Johansson $\mathrm{P}$, et al. Social support and its association with health-related quality of life among older patients with chronic heart failure. Eur J Cardiovasc Nurs 2013;12:69-77.

15. Ajrouch KJ, Antonucci TC, Janevic MR. Social networks among blacks and whites: the interaction between race and age. J Gerontol B Psychol Sci Soc Sci 2001;56:S112-S118.

16. Choi JH. Comparison of adult children's quality of relationship and care provision for elderly parents in US. U.S. and Korea, 2009.

17. Schwarz B, Albert I, Trommsdorff G, et al. Intergenerational support and life satisfaction: a comparison of chinese, indonesian, and german elderly mothers. J Cross Cult Psychol 2010;41:706-22.

18. Liu N, Zeng L, Li Z, et al. Health-related quality of life and long-term care needs among elderly individuals living alone: a cross-sectiona study in rural areas of Shaanxi Province, China. BMC Public Health 2013;13:313.

19. Roh HW, Lee Y, Lee KS, et al. Frequency of contact with noncohabitating adult children and risk of depression in elderly: a community-based three-year longitudinal study in Korea. Arch Gerontol Geriatr 2015;60:183-9.

20. Remained Elderly. "Remained elderly" care in rural in the process of labor migration. Population Journal 2006.

21. Wei L. A Comparative study on the quality of life of urban and rural elderly population in China. J of Zhejiang University 2008

22. Rabin R, de Charro F. EQ-5D: a measure of health status from the EuroQol Group. Ann Med 2001;33:337-43.

23. Liu GG, Wu H, Li M, et al. Chinese time trade-off values for EQ-5D health states. Value Health 2014;17:597-604.

24. Psaki SR, Seidman JC, Miller M, et al. Measuring socioeconomic status in multicountry studies: results from the eight-country MAL-ED study. Popul Health Metr 2014;12:8

25. Vyas S, Kumaranayake L. Constructing socio-economic status indices: how to use principal components analysis. Health Policy Plan 2006;21:459-68.

26. Krzywinski M, Altman N. Points of significance: nonparametric tests. Nat Methods 2014;11:467-8.

27. Baron RM, Kenny DA. The moderator-mediator variable distinction in social psychological research: conceptual, strategic, and statistical considerations. J Pers Soc Psychol 1986;51:1173-82.

28. Hayes AF. Beyond baron and kenny: statistical mediation analysis in the new millennium. Commun Monogr 2009;76:408-20.

29. Graziano PA, McNamara JP, Geffken GR, et al. Severity of children's ADHD symptoms and parenting stress: a multiple mediation model of self-regulation. J Abnorm Child Psychol 2011;39:1073-83.

30. Preacher KJ, Hayes AF. Asymptotic and resampling strategies for assessing and comparing indirect effects in multiple mediator models. Behav Res Methods 2008;40:879-91.

31. Sobel E, Wiley J, Sobel ME. Asymptotic Confidence Intervals for Indirect Effects. Sociological Methodology 1982;13:290-312.

32. Hayes AF. Introduction to mediation, moderation, and conditional process analysis: a regression-based approach. $J$ of Educational Measurement 2013;51:335-7.

33. Zimmer Z, Kwong J. Socioeconomic status and health among older adults in rural and urban China. J Aging Health 2004;16:44-70.

34. Liu M, Zhang Q, Lu M, et al. Rural and urban disparity in health services utilization in China. Med Care 2007;45:767-74.

35. Anderson NB, Armstead CA. Toward understanding the association of socioeconomic status and health: a new challenge for the biopsychosocial approach. Psychosom Med 1995;57:213.

36. Pol LG, Thomas RK. The demography of health and healthcare: Plenum Press, 1992:175-6.

37. Liang $Y, W u, W$. Exploratory analysis of health-related quality of life among the empty-nest elderly in rural China: an empirical study in three economically developed cities in eastern China. Health Qual Life Outcomes 2014;12:59.

38. Strine TW, Chapman DP, Balluz L, et al. Health-related quality of life and health behaviors by social and emotional support. Their relevance to psychiatry and medicine. Soc Psychiatry Psychiatr Epidemiol 2008;43:151-9.

39. Helgeson VS. Social support and quality of life. Qual Life Res 2003;12 Suppl 1:25-31.

40. He Z. Social support networks and quality of life of rural men in a context of marriage squeeze in China. Social Sciences in China 2002.

41. Guan HJ, Liu GE. Comparison analysis on health related quality of life among urban and rural residents in 4 cities of China.: Chinese Health Economics, 2015.

42. Luo J, Zhang X, Jin C, et al. Inequality of access to health care among the urban elderly in northwestern China. Health Policy 2009;93:111-7. 